\title{
Antioxidant Effects of Salidroside in the Cardiovascular System
}

\author{
Siyu Sun, ${ }^{1}$ Qinhui Tuo, ${ }^{2}$ Dongxu Li, ${ }^{1}$ Xiulong Wang, ${ }^{1}$ Xuefang Li, ${ }^{1}$ Yiyue Zhang, \\ Guoan Zhao ${ }^{(1)}{ }^{1}$ and Fei Lin $\mathbb{( D}^{1}$ \\ ${ }^{1}$ The Cardiovascular Research Center, The First Affiliated Hospital of Xinxiang Medical University, Xinxiang, \\ Henan 453003, China \\ ${ }^{2}$ The School of Pharmacy, Hunan University of Chinese Medicine, Changsha, Hunan 410208, China \\ Correspondence should be addressed to Fei Lin; linfeixixi@aliyun.com
}

Received 4 February 2020; Revised 8 May 2020; Accepted 23 May 2020; Published 26 September 2020

Academic Editor: Raffaele Capasso

Copyright $\odot 2020$ Siyu Sun et al. This is an open access article distributed under the Creative Commons Attribution License, which permits unrestricted use, distribution, and reproduction in any medium, provided the original work is properly cited.

\begin{abstract}
Cardiovascular disease is one of the main human health risks, and the incidence is increasing. Salidroside is an important bioactive component of Rhodiola rosea L., which is used to treat Alzheimer's disease, tumor, depression, and other diseases. Recent studies have shown that salidroside has therapeutic effects, to some degree, in cardiovascular diseases via an antioxidative mechanism. However, evidence-based clinical data supporting the effectiveness of salidroside in the treatment of cardiovascular diseases are limited. In this review, we discuss the effects of salidroside on cardiovascular risk factors and cardiovascular diseases and highlight potential antioxidant therapeutic strategies.
\end{abstract}

\section{Introduction}

Cardiovascular diseases (CVDs) are the most common cause of mortality globally among all diseases. Heart attack and stroke are the leading causes of CVDs-related deaths. Individuals with CVDs or with risk factors for CVDs, such as diabetes and hyperlipidemia, need early detection and appropriate medical interventions [1]. Owing to the complex mechanisms underlying CVDs, in addition to conventional medicines, the identification of natural herbal products with multiple targets is an important research goal. Salidroside (SAD) is a polyphenolic compound isolated from Rhodiola rosea L. According to Modern Practical Materia Medica, $R$. rosea L. has the following effects: (1) central inhibitory effect, (2) antifatigue effect, (3) promotion of cardiovascular function, (4) anti-inflammatory effect, (5) hypoglycemic effect, (6) antiperoxidation effect, and (7) antiradiation effect. SAD, an important bioactive component of $R$. rosea L., is effective for the treatment of Alzheimer's disease, depressive disorder, tumor, and CVDs [2-5]. In particular, accumulating studies show that SAD plays a protective role by suppressing cardiovascular risk factors and the development of coronary heart disease, heart failure, stroke, and pulmonary hypertension. This review provides a systematic overview of the effects and mechanisms of action of SAD in CVDs. In particular, we summarize evidence for the antioxidant effects of SAD, its relationships with risk factors for CVDs (e.g., obesity and aging), and its effects in various CVDs (e.g., atherosclerosis and stroke).

\section{Antioxidant Effects of SAD}

Oxidative stress refers to the imbalance between oxidation and antioxidation in vivo, and it is associated with aging and various diseases. Reactive oxygen species (ROS) overproduction is the main reason for this imbalance. Mitochondria are a major source of intracellular ROS. The antioxidant system can effectively remove the ROS produced during metabolism and protect biological macromolecules against oxidative damage by ROS, including superoxide dismutase (SOD), glutathione peroxidase (GSH-Px), and catalase (CAT) $[6,7]$. Accordingly, mitochondrial damage or a decrease in antioxidant enzyme synthesis results in excessive ROS production. $R$. rosea L. ethanol extract, which has high levels of phenolic compounds, particularly SAD, has strong antioxidant activity, as determined by DPPH, ABTS, and 
FRAP assays [8]. Further studies have shown that SAD not only inhibits ROS production by the regulation of mitochondrial biogenesis but also inhibits ROS-mediated CVDs by increasing the activity of the antioxidant enzymes SOD and GSH-Px $[9,10]$. Finally, SAD has antioxidant effects in vivo and in vitro.

\section{Cardiovascular Risk Factors}

CVDs are the result of long-term interactions among many adverse factors. Risk factors include hyperlipidemia, smoking, drink, diabetes, obesity, aging, and genetic factors. Active prevention and treatment of these risk factors are essential to reduce the incidence of CVDs. Extensive research has shown that SAD influences cardiovascular risk factors, as summarized below.

3.1. Obesity and Diabetes. Epidemiological analyses have proven a clear relationship among diabetes, obesity, and CVDs. Notably, the progression from obesity to diabetes associated with an abnormal lipid profile is strongly correlated with insulin resistance. As highlighted in the literature, the relationships among obesity, abnormal lipid profiles, insulin resistance, and diabetes contribute to the occurrence and development of CVDs [11, 12]. Various studies have evaluated the use of SAD for the treatment of obesity, insulin resistance, and diabetes. Wang et al. find that $50 \mathrm{mg} / \mathrm{kg} /$ day SAD for 48 days can significantly repress the elevation of body weight and adipogenesis in epididymal white adipose tissues by reducing food intake. It also attenuates the levels of triglycerides and total cholesterol in the liver. SAD can decrease the levels of total triacylglycerides, total cholesterol, low-density lipoprotein cholesterol, and high-density lipoprotein cholesterol in the plasma [13, 14]. Insulin resistance is involved in the pathogenesis of these disorders and may benefit from intervention with SAD. As expected, SAD reduces blood glucose and serum insulin levels and increases sensitivity to insulin. These effects may be mediated by AMPK/SIRT1 signaling and the mitochondria-related AMPK/PI3K/Akt/ GSK3 $\beta$ pathway $[15,16]$. Insulin resistance is responsible for the development of type 2 diabetes mellitus. Recent studies have shown that SAD stimulates glucose uptake, regulates hepatic gluconeogenesis and lipid metabolism, and improves $\beta$-cell survival in the treatment of diabetes $[17,18]$.

SAD has beneficial effects on vascular diastolic dysfunction via the soluble guanylyl cyclase pathway in the Goto-Kakizaki model of type 2 diabetes [19]. SAD $(1-10 \mu \mathrm{g} /$ $\mathrm{ml})$ has a protective effect on high glucose-induced endothelial cell injury by activating the $\mathrm{Ca} / \mathrm{CaM} / \mathrm{CAMKII} / \mathrm{eNOS}$ pathway [20]. SAD prevents advanced glycation endproduct-induced endothelial dysfunction, and its effects may be attributed, in part, to the induction of $\mathrm{HO}-1$ and the attenuation of phosphorylated NF-kB p65 [21]. In addition, SAD ameliorates diabetic nephropathy and antihyperalgesic activity and prevents cognitive impairment in a rat model of diabetes [22-24]. Accordingly, we expect SAD to be useful for the treatment of lifestyle-related diseases, such as hyperlipidemia, exogenous obesity, and diabetes.

3.2. Hyperlipidemia. Hyperlipidemia is a risk factor for CVDs. A large number of studies have proven that the interplay between lipids and immune cell infiltration is the main cause of the formation of vascular atherosclerosis (AS). AS begins with the accumulation and oxidation of subendothelial cholesterol in blood vessels. This subsequently stimulates the production of innate and acquired immunity and causes chronic inflammation of the vascular wall [25]. Since the theory of oxidative modification of LDL in AS was initially proposed 30 years ago, oxidized low-density lipoprotein (ox-LDL) has been used as a stimulator of AS. The protective effects of SAD have been demonstrated in oxLDL-injured HUVECs, and these effects may be related to the inhibition of oxidative stress inhibition and restoration of mitochondrial dysfunction by activating the AMPK/ SIRT1 pathway [26]. Further research has shown that SAD also protects against ox-LDL-induced endothelial injury by promoting autophagy via the SIRT1-FoxO1 signaling pathway [27].

3.3. Nonalcoholic Fatty Liver Disease. Increasing studies indicate that nonalcoholic fatty liver disease (NAFLD) is independently associated with the development of carotid intima-media thickening and plaques and coronary artery calcification, with a $65 \%$ increase in CVDs events [28]. Consistent with the effects of SAD on obesity, lipid metabolism, insulin resistance, and diabetes, it has a regulatory effect on high-fat diet-induced NAFLD. In particular, SAD has an anti-NAFLD effect via the inhibition TRPM2 ion channel activation, regulation of AMPK-dependent TXNIP/ NLRP3 pathways, insulin signaling pathway, and gut microbiota-bile acid-farnesoid X receptor axis [29-32].

3.4. Aging. It is well-known that aging is one of the key factors in the occurrence of CVDs. Antioxidants have beneficial effects in aging-associated CVDs [33]. Recently, the Chinese medicinal herb Rhodiola has been reported to have antiaging activity in Alzheimer's disease, Parkinson's disease, CVDs, and so on [5]. SAD, as its main component with antioxidant effects, prolongs the lifespan and delays the onset of age-related biomarkers [34]. In addition, SAD attenuates endothelial cellular senescence and inhibits the vascular aging response [35].

\section{Cardiovascular Diseases}

\subsection{Coronary Heart Disease}

4.1.1. Atherosclerosis. AS is the main pathological basis of coronary heart disease, cerebral atherosclerotic infarction, and peripheral vascular disease. The pathogenesis of AS involves several key factors: (1) endothelial cell injury, (2) lipid accumulation at the injury site, (3) internalization of lipids by monocytes and foam cell formation, and (4) inflammatory factor-induced proliferation of vascular smooth 
muscle cells. The protective effects of SAD have been investigated in female LDL receptor knockout $\left(\mathrm{LDLr}^{-/}\right)$mice treated with a high-fat diet to induce AS. SAD treatment $(50 \mathrm{mg} / \mathrm{kg} / \mathrm{day}$ for 8 weeks) clearly reduced the plaque area of the aortic arch by lowering lipids and anti-inflammatory effects [36]. Furthermore, NLRP3-related pyroptosis might be another mechanism by which SAD decreases AS plaque formation [37]. The antiatherosclerotic effects of SAD protect endothelial function by promoting nitric oxide (NO) production, which is associated with mitochondria depolarization and the subsequent activation of the AMPK/PI3K/ Akt/eNOS pathway [38].

Endothelial dysfunction plays important roles in CVDs, including AS. Oxidative stress, the renin-angiotensin system, ox-LDL, and homocysteine are the main causes of endothelial injuries [39]. Consequently, endothelial cells could be targets of SAD to protect against AS. Pretreatment of cells with SAD significantly reduces endothelial injury in a process mediated by the regulation of oxidation stress signaling pathways, such as the AMPK pathway, mTOR pathway, and Nrf2 pathway, in response to stimulation by hydrogen peroxide $\left(\mathrm{H}_{2} \mathrm{O}_{2}\right)$ [40-42]. Using another model of homocysteine stimulation, SAD improved NO bioavailability, stimulated mitochondrial biogenesis, inhibited ROS production, and regulated the ER-stress pathway to protect endothelial cell function $[9,43,44]$. In addition, SAD exerts angiogenic and cytoprotective effects via the Akt/mTOR/ p70S6K and MAPK signaling pathways in human bone marrow-derived endothelial progenitor cells [45].

4.1.2. Myocardial Ischemia. Myocardial infraction (MI) is a CVDs caused by persistent ischemia and hypoxia of the coronary artery. Ischemia/reperfusion (I/R) injury is an important complication during the treatment of $\mathrm{MI}$ in clinical settings. However, effective cardioprotective therapies are lacking for MI and I/R injury. Some scholars have recently proposed that focusing on the rational combination of judiciously selected and multitargeted therapies may be effective [46]. Multitarget mechanisms underlying the effects of SAD under MI have been identified. Recent studies have shown that SAD is a multitarget drug. On the one hand, SAD protects against LPS-induced myocardial injury in vivo and hydrogen peroxide-induced myocardial cell injury in vitro via the activation of the PI3K/Akt pathway $[47,48]$. On the other hand, SAD protects against hypoxia-induced myocardial cell death and promotes cardiac angiogenesis in acute MI rats by upregulating HIF- $1 \alpha$ and the VEGF-mediated pathway $[49,50]$. In addition, SAD inhibits apoptosis by restoring the tricarboxylic acid cycle and by the preservation of mitochondrial integrity $[51,52]$. Finally, SAD attenuates isoproterenol-induced acute MI by the regulation of the Nox/NF- $\kappa$ B/AP1 pathway [53]. These studies support the role of SAD as a potential treatment for ischemic heart disease.

I/R injury refers to ischemia and subsequent reperfusion injury in acute MI. Recently, the effects and mechanisms of action of traditional Chinese medicine on I/R injury have been gradually characterized [54]. SAD is effective in preventing I/R injury. By pretreating rats with $50 \mathrm{mg} / \mathrm{kg}$ $\mathrm{SAD}, \mathrm{Xu}$ et al. found that SAD activates the PI3K/Akt pathway and reduces apoptosis in cardiomyocytes, which in turn inhibits I/R injury [55]. In cell experiments, myocardial cells were induced by hypoxia/reoxygenation to mimic I/R injury, and the injury effect was inhibited by SAD pretreatment, as evidenced by the suppression of apoptosis, an increase in $\mathrm{N}$-acetylglucosamine linkage to cellular proteins, and the activation of Akt signaling [56-58].

4.2. Stroke. Similar to MI, increasing evidence shows that $\mathrm{SAD}$ and its analogues reduce ischemic stroke and cerebral I/ $\mathrm{R}$ injury in adult rats. In general, the mechanisms underlying the neuroprotective effects of SAD may involve three pathways. First, SAD inhibits the inflammatory responses in multiple ischemic stroke processes, as indicated by the reduction of LPS-induced BV2 microglial cell mobility via NF-B and MAPK signaling, the reduction of inflammatory effects via PI3K/Akt signaling after permanent middle cerebral artery occlusion or cerebral I/R injury, and the induction of primary microglia from the M1 phenotype to M2 phenotype [59-62]. Second, protective effects of SAD against ischemic stroke are related to endothelial function. The intraperitoneal administration of SAD before middle cerebral artery occlusion improves human brain microvascular endothelial cell activity by activating the PI3K/Akt pathway. Furthermore, SAD alleviates brain ischemic injury, and I/R injury caused bloodbrain barrier injury by delayed tPA treatment and the inhibition of tumor necrosis factor-alpha $[63,64]$. Third, SAD regulates mitochondrial function against exertional heat stroke-induced organ damage in a rat model [65]. Recently, increasing SAD analogues have been found to have the same neuroprotective effects. For example, 2-(4-methoxyphenyl) ethyl-2-acetamido-2-deoxy- $\beta$-D-pyranoside (GlcNAc-Sal) confers neuroprotective effects via the regulation of local glucose metabolism by increasing glucose uptake, $O$ GlcNAcylation, and elevating GLUT3 expression in a rat model of cerebral ischemic injury [66-68]. Further research has shown that GlcNAc-Sal prevents brain I/R injury and suppresses mouse hippocampal HT22 cell apoptosis by reducing ROS generation, NO production, and the expression of caspase-3 [69]. In addition, the SAD metabolite $p$-tyrosol at a dose of $20 \mathrm{mg} / \mathrm{kg}$ also attenuates neuronal damage in the hippocampus as well as lipid peroxidation in brain tissue in animals subjected to global cerebral ischemia with reperfusion [70]. Taken together, SAD could prevent stroke and exert neuroprotective effects via multiple mechanisms.

4.3. Cardiac Function Injury in Exhaustive Exercise. Exercise training can maintain health, but exhausting exercise leads to a reduction in cardiac function. There is experimental evidence that SAD has a protective effect against exercise-induced decreases in cardiac function. The protective effects of SAD have been investigated in rats trained with exhaustive swimming. SAD treatment evidently decreases the levels of brain natriuretic peptide, cardiac troponin I, and ROS in the serum [71]. It also decreases the level of CK-MB, which is mainly used to diagnose acute 


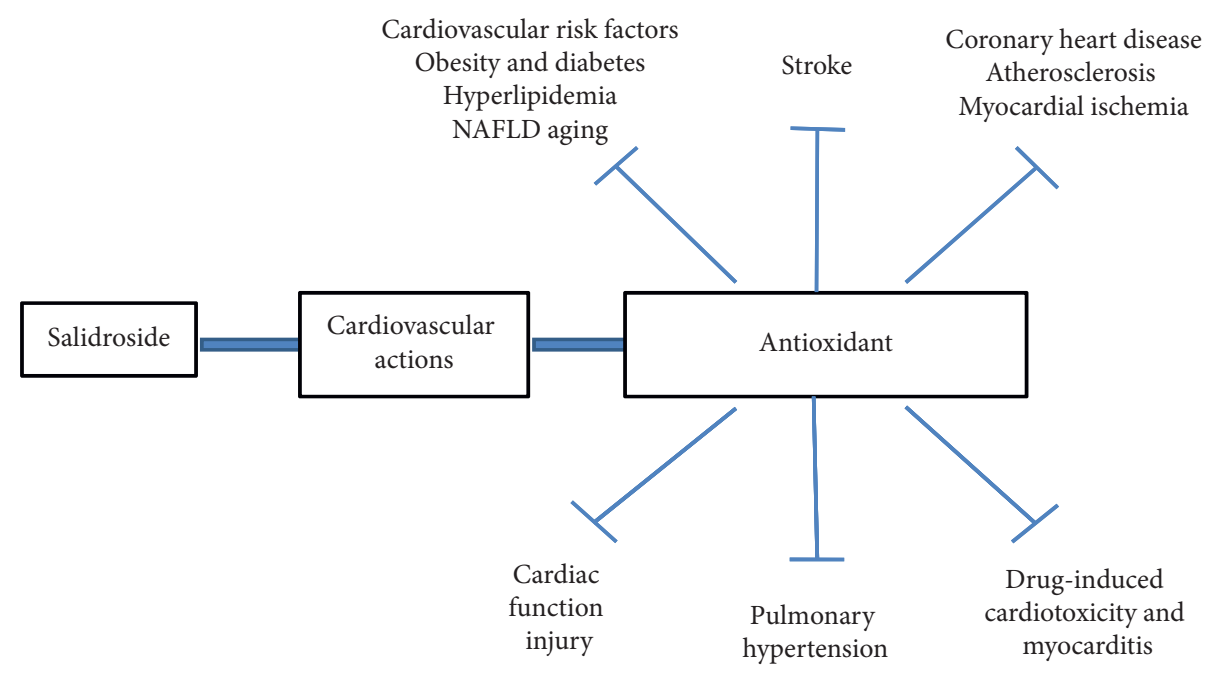

FIgURE 1: Cardiovascular actions of salidroside.

myocardial injury [72]. Further research has shown that the underlying mechanisms include antioxidative stress by MAPK signal transduction and improved mitochondrial respiratory function by the Nrf2 signaling pathway $[71,72]$.

4.4. Pulmonary Hypertension. Pulmonary hypertension refers to a hemodynamic and pathophysiological state in which pulmonary arterial pressure increases beyond a certain threshold, leading to right heart failure. Pulmonary hypertension is a common and frequently occurring disease with high rates of morbidity and mortality. Increasing evidence shows that Rhodiola may be used for the treatment of pulmonary hypertension [73]. The abnormal proliferation and apoptotic resistance of pulmonary vascular smooth muscle cells are major causes of pulmonary hypertension. Rhodiola and SAD have antipulmonary hypertension effects, mainly by inhibiting cell proliferation and promoting cell apoptosis. The main mechanisms include the regulation of the adenosine A2a receptor-related mitochondria-dependent apoptosis pathway, AMPK pathway, and AKT pathway [74-76]. In addition, SAD can also reduce blood pressure in diabetic rats and has a dual protective role in diabetes and hypertension [77].

4.5. Drug-Induced Cardiotoxicity and Myocarditis. Anthraquinones are widely used for the treatment of acute and chronic leukemia, malignant lymphoma, breast cancer, and other solid tumors. However, their clinical applications are limited by cardiotoxicity. SAD inhibits this cardiotoxicity. Wang et al. found that SAD effectively protects cardiomyocytes against doxorubicin-induced cardiotoxicity by suppressing excessive oxidative stress and activating a $\mathrm{Bcl} 2$ mediated survival signaling pathway [78]. Zhang et al. found that SAD has a protective effect against epirubicin-induced early left ventricular regional systolic dysfunction in patients with breast cancer [79].

Myocarditis is often caused by the immune response after viral infection. Wang et al. found that SAD possesses antiviral activity in vivo and in vitro by an antioxidant effect and the inhibition of cytokine expression [80]. Similarly, SAD reduces LPS-induced myocardial depression in sepsis by regulating the inflammatory response [81]. Therefore, it is a candidate therapeutic agent for myocarditis.

\section{Molecular Targets of SAD in CVDs}

In light of emerging evidence for the cardiovascular benefits of SAD (Figure 1), we discuss the major molecular targets in detail (Figure 2). (1) AMPK signaling pathway. AMPK plays a major role in regulating the cellular energy balance. The occurrence and development of CVDs are closely related to the disruption of cellular energy metabolism. Therefore, AMPK is an important molecular determinant of CVDs [82]. In a review of recent literature, Zheng concluded that AMPK is an important target of SAD in diabetes [17]. Furthermore, AMPK can regulate autophagy, mitochondrial function, apoptosis, and inflammatory responses by interacting with PI3K/Akt, mTOR, and SIRT1 in endothelial cells $[26,38,40]$. (2) PI3K/Akt signaling pathway. Akt can widely regulate cell proliferation, survival, growth, migration, and other physiological functions. SAD activation of the PI3K/ Akt signaling pathway and downstream targets, such as mTOR and GSK $3 \beta$, determines its function in cardiovascular processes $[15,45]$. (3) Mitochondria-dependent signaling pathway. Traditional Chinese medicine used to treat CVDs is closely related to the regulation of mitochondrial function [83]. SAD results in specific mitochondrial depolarization and therefore is able to potently regulate the mitochondria-dependent signaling pathway, modulate mitochondrial-mediated apoptosis, and decrease ROS production [38, 52]. (4) Nrf2 signaling pathway. Nrf2 is an important transcription factor in the regulation of oxidative stress and a central regulator in the maintenance of intracellular redox homeostasis [84]. SAD, as an antioxidant, can induce Nrf2 nuclear translocation, activate the expression of Nrf2-regulated antioxidant enzyme genes, and decrease the levels of intercellular ROS [42]. 


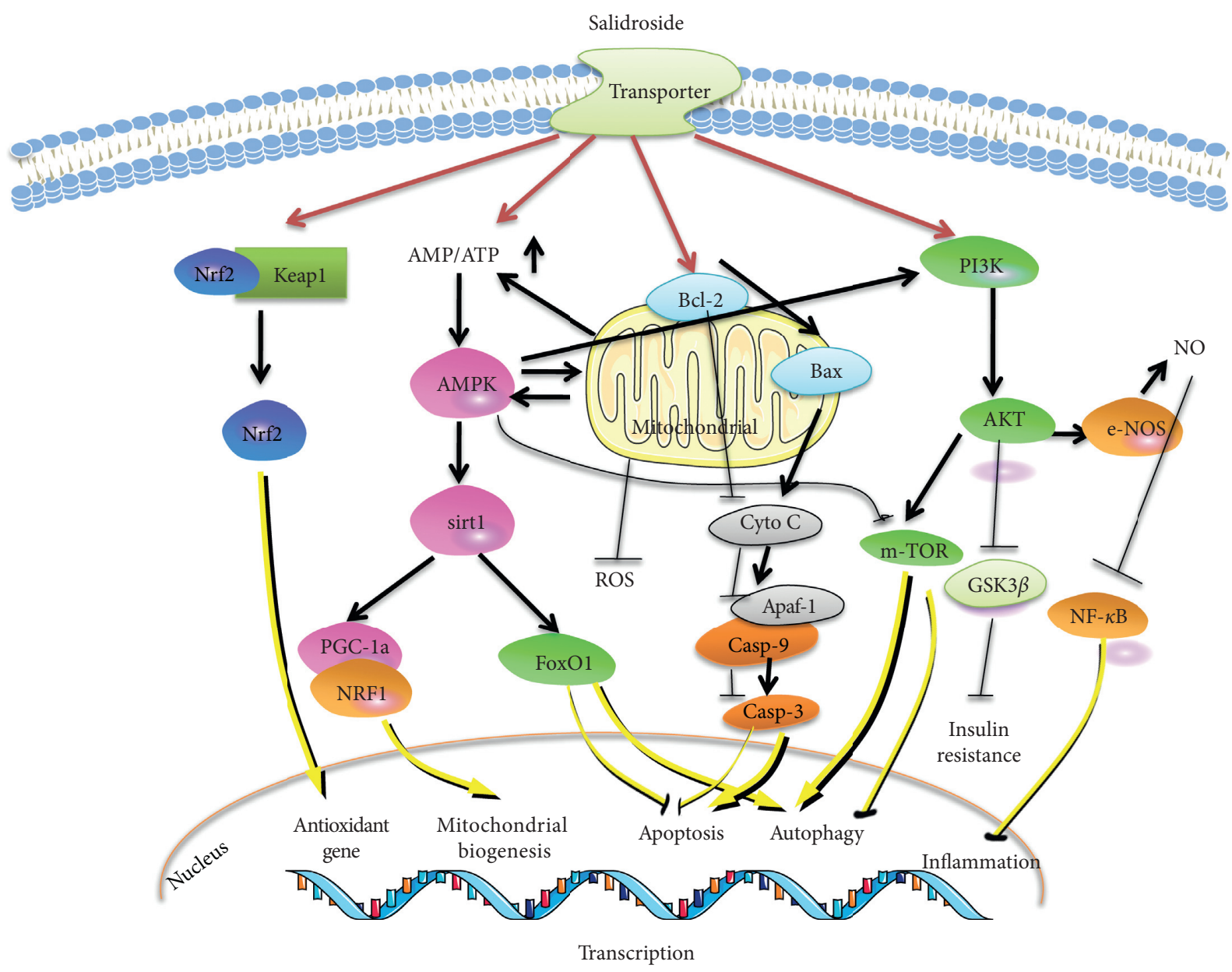

Figure 2: Molecular targets of salidroside.

\section{Discussion}

Based on the research summarized in this review, we conclude that SAD has an anti-CVDs effect via an antioxidative mechanism. However, the clinical use of SAD requires additional experimental studies. For example, the bioavailability of SAD is not clear. Guo et al. have reported that $\mathrm{SAD}$ is rapidly metabolized to $p$-tyrosol after i.v. administration $(50 \mathrm{mg} / \mathrm{kg})$ in rats and has the highest concentration in the heart. However, $64.00 \%$ of the total dose is excreted through the urine in the form of SAD [85]. Owing to the lack of detailed bioavailability, pharmacology, and toxicology experiments, an appropriate clinically recommended dose has not been established, limiting its clinical applications. Additionally, SAD may have effects on cardiovascular drug metabolism. The main enzymes for metabolizing drugs in humans are cytochrome $\mathrm{P} 450$ proteins (CYP). R. rosea L. inhibits CYP2C9 [86]. Losartan, glimepiride, and other drugs commonly used to treat hypertension and diabetes are mainly metabolized by CYP2C9 enzymes. The inhibition of these metabolic enzymes may increase the long-term efficacy of drugs. However, it may also increase hepatotoxicity. Finally, there are few clinical trials of SAD in CVDs. Although Dazhu Hongjingtian injection (YBZ11852006) promotes blood circulation and reduces blood stasis, it is mainly used for the treatment of stable exertion angina pectoris in coronary heart disease. Traditional Chinese medicine syndrome differentiation for heart blood stasis is based on the following symptoms: chest pain, colic, immovable, pain-induced shoulder back and medial arm, chest tightness, palpitation restlessness, dark lips and tongue, and fine pulse. However, to confirm that SAD is the main component underlying its functions, more experimental research is needed.

\section{Conclusion}

CVDs are the result of the long-term accumulation of multiple factors. Therefore, it is necessary to develop multitargeted treatments. Overall, salidroside has an established chemical structure and metabolites with an efficient heart-targeting effect. It also affects cardiovascular diseases via anti-inflammatory, antioxidant, and antiapoptotic effects, including atherosclerotic coronary heart disease, MI, and stroke. Therefore, SAD is a potential anticardiovascular drug. However, in view of the lack of clarity regarding its mechanism of action, additional experimental research is needed for clinical applications. 


\section{Conflicts of Interest}

The authors declare that there are no conflicts of interest regarding the publication of this paper.

\section{Acknowledgments}

This study was supported by the National Natural Science Foundation of China (nos. 81503421 and 81673722), Special Funds for Funding Funds of Fundamental Research Business Fees of Central Public Welfare Research Institutions (no. JDZX2015248), Key Scientific Research Projects of Higher Education Institutions in Henan Province (nos. 18A320005 and 19A360032), and Young Elite Scientists Sponsorship Program by CAST (YESS, 2017QNRC001).

\section{References}

[1] M. A. Lambert and J. J. F. Belch, "Medical management of critical limb ischaemia: where do we stand today?" Journal of Internal Medicine, vol. 274, no. 4, pp. 295-307, 2013.

[2] Z. Zhifeng, H. Jing, Z. Jizhou, X. Qing, H. Juan, and C. Lidian, "Pharmacological activities, mechanisms of action, and safety of salidroside in the central nervous system," Drug Design, Development and Therapy, vol. 12, pp. 1479-1489, 2018.

[3] J. D. Amsterdam and A. G. Panossian, "Rhodiola rosea L. as a putative botanical antidepressant," Phytomedicine, vol. 23, no. 7, pp. 770-783, 2016.

[4] M. Hosein Farzaei, R. Bahramsoltani, and R. Rahimi, "Phytochemicals as adjunctive with conventional anticancer therapies," Current Pharmaceutical Design, vol. 22, no. 27, pp. 4201-4218, 2016.

[5] W. Zhuang, L. Yue, X. Dang et al., "Rosenroot (rhodiola): potential applications in aging-related diseases," Aging and Disease, vol. 10, no. 1, pp. 134-146, 2019.

[6] A. O. Chen and M. A. Birch-Machin, "Mitochondria-targeted antioxidants," The FASEB Journal, vol. 29, no. 12, pp. 4766-4771, 2015.

[7] H. N. Siti, Y. Kamisah, and J. Kamsiah, "The role of oxidative stress, antioxidants and vascular inflammation in cardiovascular disease (a review)," Vascular Pharmacology, vol. 71, pp. 40-56, 2015.

[8] O. Kosakowska, K. Bączek, J. L. Przybył et al., “Antioxidant and antibacterial activity of roseroot (Rhodiola rosea L.) dry extracts," Molecules, vol. 23, no. 7, p. 1767, 2018.

[9] S. Xing, X. Yang, W. Li et al., "Salidroside stimulates mitochondrial biogenesis and protects against $\mathrm{H}_{2} \mathrm{O}_{2}$-induced endothelial dysfunction," Oxidative Medicine and Cellular Longevity, vol. 2014, Article ID 904834, 13 pages, 2014.

[10] M. H. Li, C. F. Tang, and J. Q. Ouyang, "Influence of salidroside from Rhodiola sachalinensis A. bor on some related indexes of free radical and energy metabolism after exercise in mice," Zhongguo Ying Yong Sheng Li Xue Za Zhi, vol. 28, no. 1, pp. 53-56, 2012.

[11] V. T. Samuel, K. F. Petersen, and G. I. Shulman, "Lipid-induced insulin resistance: unravelling the mechanism," The Lancet, vol. 375, no. 9733, pp. 2267-2277, 2010.

[12] A. Golay and J. Ybarra, "Link between obesity and type 2 diabetes," Best Practice \& Research Clinical Endocrinology \& Metabolism, vol. 19, no. 4, pp. 649-663, 2005.

[13] M. Wang, L. Luo, L. Yao et al., "Salidroside improves glucose homeostasis in obese mice by repressing inflammation in white adipose tissues and improving leptin sensitivity in hypothalamus," Scientific Reports, vol. 6, no. 1, p. 25399, 2016.

[14] X.-R. Zhang, X.-J. Fu, D.-S. Zhu et al., "Salidroside-regulated lipid metabolism with down-regulation of miR-370 in type 2 diabetic mice," European Journal of Pharmacology, vol. 779, pp. 46-52, 2016.

[15] T. Zhang, X. Yang, D. Wu et al., "Salidroside ameliorates insulin resistance through activation of a mitochondria-associated AMPK/PI3K/Akt/GSK3 $\beta$ pathway," British Journal of Pharmacology, vol. 172, no. 13, pp. 3284-3301, 2015.

[16] B. Xing, Y. Dun, W. Zhang et al., "Anti-insulin resistance effects of salidroside through mitochondrial quality control," Journal of Endocrinology, vol. 244, no. 2, pp. 383-393, 2019.

[17] T. Zheng, F. Bian, L. Chen, Q. Wang, and S. Jin, "Beneficial effects of Rhodiola and salidroside in diabetes: potential role of AMP-activated protein kinase," Molecular Diagnosis \& Therapy, vol. 23, no. 4, pp. 489-498, 2019.

[18] L. Ju, X. Wen, C. Wang et al., "Salidroside, a natural antioxidant, improves $\beta$-cell survival and function via activating AMPK pathway," Frontiers in Pharmacology, vol. 8, p. 749, 2017.

[19] A. Alameddine, Z. Fajloun, J. Bourreau et al., "The cardiovascular effects of salidroside in the Goto-Kakizaki diabetic rat model," Journal of Physiology and Pharmacology: An Official Journal of the Polish Physiological Society, vol. 66, no. 2, pp. 249-257, 2015.

[20] Z. Chen and $\mathrm{X}$. Wu, "Salidroside attenuates high glucoseinduced apoptosis in human umbilical vein endothelial cells via activating the $\mathrm{Ca}(2)+/ \mathrm{CaM} / \mathrm{CAMKII} \delta /$ NOS pathway," Zhonghua Xin Xue Guan Bing Za Zhi, vol. 42, no. 4, pp. 327-333, 2014.

[21] P. Zhang, R. Y. Li, and W. Zang, "Salidroside protects against advanced glycation end products-induced vascular endothelial dysfunction," Medical Science Monitor, vol. 24, pp. 2420-2428, 2018.

[22] M. Déciga-Campos, M. E. González-Trujano, R. VenturaMartínez et al., "Antihyperalgesic activity of Rhodiola r osea in a diabetic rat model," Drug Development Research, vol. 77, no. 1, pp. 29-36, 2016.

[23] X. Hao, J. Yuan, and H. Dong, "Salidroside prevents diabetesinduced cognitive impairment via regulating the Rho pathway," Molecular Medicine Reports, vol. 19, no. 1, pp. 678-684, 2019.

[24] H. Xue, P. Li, Y. Luo et al., "Salidroside stimulates the Sirt1/ PGC- $1 \alpha$ axis and ameliorates diabetic nephropathy in mice," Phytomedicine, vol. 54, pp. 240-247, 2018.

[25] M. Lacy, D. Atzler, R. Liu, M. de Winther, C. Weber, and E. Lutgens, "Interactions between dyslipidemia and the immune system and their relevance as putative therapeutic targets in atherosclerosis," Pharmacology \& Therapeutics, vol. 193, pp. 50-62, 2019.

[26] D. de Winther, X. Sun, S. Lv et al., "Salidroside attenuates oxidized low-density lipoprotein-induced endothelial cell injury via promotion of the AMPK/SIRT1 pathway," International Journal of Molecular Medicine, vol. 43, no. 6, pp. 2279-2290, 2019.

[27] Z. Zhu, J. Li, and X. Zhang, "Salidroside protects against oxLDL-induced endothelial injury by enhancing autophagy mediated by SIRT1-FoxO1 pathway," BMC Complementary Alternative Medicine, vol. 19, no. 1, pp. 111-121, 2019.

[28] A. Leonardo, F. Nascimbeni, A. Mantovani, and G. Targher, "Hypertension, diabetes, atherosclerosis and NASH: cause or 
consequence?" Journal of Hepatology, vol. 68, no. 2, pp. 335-352, 2017.

[29] Q. Feng, C. Liu, W. Gao, X.-l. Geng, and N. Dai, "Salidrosidemitigated inflammatory injury of hepatocytes with non-alcoholic fatty liver disease via inhibition TRPM2 ion channel activation," Diabetes, Metabolic Syndrome and Obesity: Targets and Therapy, vol. 12, pp. 2755-2763, 2019.

[30] T. Zheng, X. Yang, W. Li et al., "Salidroside attenuates highfat diet-induced nonalcoholic fatty liver disease via AMPKdependent TXNIP/NLRP3 pathway," Oxidative Medicine and Cellular Longevity, vol. 2018, Article ID 8597897, 17 pages, 2018.

[31] H. Li, H. Ying, A. Hu, D. Li, and Y. Hu, "Salidroside modulates insulin signaling in a rat model of nonalcoholic steatohepatitis," Evidence-Based Complementary and Alternative Medicine, vol. 2017, Article ID 9651371, 6 pages, 2017.

[32] H. Li, Y. Xi, X. Xin et al., "Salidroside improves high-fat dietinduced non-alcoholic steatohepatitis by regulating the gut microbiota-bile acid-farnesoid X receptor axis," Biomedicine \& Pharmacotherapy, vol. 124, Article ID 109915, 2020.

[33] M. Abdellatif, S. Sedej, D. Carmona-Gutierrez et al., "Autophagy in cardiovascular aging," Circulation Research, vol. 123, no. 7, pp. 803-824, 2018.

[34] X. Wang, X. Du, Y. Zhou, S. Wang, F. Su, and S. Zhang, "Time-dependent effects of late-onset dietary intake of salidroside on lifespan and age-related biomarkers of the annual fish Nothobranchius guentheri," Oncotarget, vol. 9, no. 19, pp. 14882-14894, 2018.

[35] S. S. Xing, J. Li, L. Chen et al., "Salidroside attenuates endothelial cellular senescence via decreasing the expression of inflammatory cytokines and increasing the expression of SIRT3," Mechanisms of Ageing and Development, vol. 175, pp. 1-6, 2018.

[36] B.-C. Zhang, W.-M. Li, R. Guo, and Y.-W. Xu, "Salidroside decreases atherosclerotic plaque formation in low-density lipoprotein receptor-deficient mice," Evidence-Based Complementary and Alternative Medicine, vol. 2012, Article ID 607508, 5 pages, 2012.

[37] S. S. Xing, J. Yang, W. J. Li et al., "Salidroside decreases atherosclerosis plaque formation via inhibiting endothelial cell pyroptosis," Inflammation, vol. 43, no. 2, pp. 433-440, 2020.

[38] S. S. Xing, X. Y. Yang, T. Zheng et al., "Salidroside improves endothelial function and alleviates atherosclerosis by activating a mitochondria-related AMPK/PI3K/Akt/eNOS pathway," Vascular Pharmacology, vol. 72, pp. 141-152, 2015.

[39] H. Haybar, S. Shahrabi, H. Rezaeeyan, R. Shirzad, and N. Saki, "Endothelial cells: from dysfunction mechanism to pharmacological effect in cardiovascular disease," Cardiovascular Toxicology, vol. 19, no. 1, pp. 13-22, 2019.

[40] X. T. Zheng, Z. H. Wu, Y. Wei et al., "Induction of autophagy by salidroside through the AMPK-mTOR pathway protects vascular endothelial cells from oxidative stress-induced apoptosis," Molecular and Cellular Biochemistry, vol. 425, no. 12, pp. 125-138, 2017.

[41] M.-C. Xu, H.-M. Shi, H. Wang, and X.-F. Gao, "Salidroside protects against hydrogen peroxide-induced injury in HUVECs via the regulation of REDD1 and mTOR activation," Molecular Medicine Reports, vol. 8, no. 1, pp. 147-153, 2013.

[42] Z. Yao, Z. Ya-Jie, L. Wei-Wei, S. Ai-Wu, and G. Ning, "Salidroside suppresses HUVECs cell injury induced by oxidative stress through activating the Nrf2 signaling pathway," Molecules, vol. 21, no. 8, p. 1033, 2016.
[43] S. B. Leung, H. Zhang, C. W. Lau, Y. Huang, and Z. Lin, "Salidroside improves homocysteine-induced endothelial dysfunction by reducing oxidative stress," Evidence-Based Complementary and Alternative Medicine, vol. 2013, Article ID 679635, 8 pages, 2013.

[44] L. Zhu, F. Jia, J. Wei et al., "Salidroside protects against homocysteine-induced injury in HUVECs via the regulation of endoplasmic reticulum stress," Cardiovascular Therapeutics, vol. 35, no. 1, pp. 33-39, 2016.

[45] Y. Tang, C. Vater, A. Jacobi, C. Liebers, X. Zou, and M. Stiehler, "Salidroside exerts angiogenic and cytoprotectiveeffects on human bone marrow-derived endothelial progenitor cells via Akt/mTOR/p70S6K and MAPK signalling pathways," British Journal of Pharmacology, vol. 171, no. 9, pp. 2440-2456, 2014.

[46] S. M. Davidson, P. Ferdinandy, I. Andreadou et al., "Multitarget strategies to reduce myocardial is chemia/reperfusion injury," Journal of the American College of Cardiology, vol. 73, no. 1, pp. 89-99, 2019.

[47] Y. Zhu, Y. P. Shi, D. Wu et al., "Salidroside protects against hydrogen peroxide-induced injury in cardiac H9c2 cells via PI3K-Akt dependent pathway," DNA and Cell Biology, vol. 30, no. 10, pp. 809-819, 2011.

[48] L. Chen, L. Peng, C. Ma, X. Feng et al., "Salidroside suppressing LPS-induced myocardial injury by inhibiting ROSmediated PI3K/Akt/mTOR pathway in vitro and in vivo," Journal of Cellular and Molecular Medicine, vol. 21, no. 12, pp. 3178-3189, 2017.

[49] J. Zhang, A. Liu, R. Hou et al., "Salidroside protects cardiomyocyte against hypoxia-induced death: a HIF- $1 \alpha$-activated and VEGF-mediated pathway," European Journal of Pharmacology, vol. 607, no. 1-3, pp. 6-14, 2009.

[50] X. F. Gao, H. M. Shi, T. Sun, and H. Ao, "Effects of radix et rhizoma Rhodiolae Kirilowii on expressions of von Willebrand factor, hypoxia-inducible factor 1 and vascular endothelial growth factor in myocardium of rats with acute myocardial infarction," Journal of Chinese Integrative Medicine, vol. 7, no. 5, pp. 434-440, 2009.

[51] Z. W. Xu, X. Chen, X. H. Jin et al., "SILAC-based proteomic analysis reveals that salidroside antagonizes cobalt chlorideinduced hypoxic effects by restoring the tricarboxylic acid cycle in cardiomyocytes," Journal of Proteomics, vol. 130, pp. 211-220, 2016.

[52] H. Zhong, H. Xin, L.-X. Wu, and Y.-Z. Zhu, "Salidroside attenuates apoptosis in ischemic cardiomyocytes: a mechanism through a mitochondria-dependent pathway," Journal of Pharmacological Sciences, vol. 114, no. 4, pp. 399-408, 2010.

[53] L. Zhu, T. Wei, X. Chang et al., "Effects of salidroside on myocardial injury in vivo in vitrovia regulation of Nox/NF$\kappa \mathrm{B} / \mathrm{AP} 1$ pathway," Inflammation, vol. 38 , no. 4, pp. 1589-1598, 2015.

[54] J.-Y. Han, Q. Li, Z.-Z. Ma, and J.-Y. Fan, "Effects and mechanisms of compound Chinese medicine and major ingredients on microcirculatory dysfunction and organ injury induced by ischemia/reperfusion," Pharmacolology \& Therapeutics, vol. 177, pp. 146-173, 2017.

[55] M. C. Xu, H. M. Shi, X. F. Gao, and H. Wang, "Salidroside attenuates myocardial ischemia-reperfusion injury via PI3K/ Akt signaling pathway," Journal of Asian Natural Products Research, vol. 15, no. 3, pp. 244-252, 2013.

[56] M. Y. Sun, D. S. Ma, S. Zhao, L. Wang, C.-Y. Ma, and Y. Bai, "Salidroside mitigates hypoxia/reoxygenation injury by alleviating endoplasmic reticulum stress-induced apoptosis in 
H9c2 cardiomyocytes," Molecular Medicine Reports, vol. 18, no. 4, pp. 3760-3768, 2018.

[57] T. Wu, H. Zhou, Z. Jin et al., "Cardioprotection of salidroside from ischemia/reperfusion injury by increasing $\mathrm{N}$-acetylglucosamine linkage to cellular proteins," European Journal of Pharmacology, vol. 613, no. 1-3, pp. 93-99, 2009.

[58] K. Zheng, Z. Sheng, Y. Li, and H. Lu, "Salidroside inhibits oxygen glucose deprivation (OGD)/re-oxygenation-induced H9c2 cell necrosis through activating of Akt-Nrf2 signaling," Biochemical and Biophysical Research Communications, vol. 451, no. 1, pp. 79-85, 2014.

[59] Y. Wei, H. Hong, X. Zhang et al., "Salidroside inhibits inflammation through PI3K/Akt/HIF signaling after focal cerebral ischemia in rats," Inflammation, vol. 40, no. 4, pp. 1-13, 2017.

[60] X. Liu, S. Wen, F. Yan et al., "Salidroside provides neuroprotection by modulating microglial polarization after cerebral ischemia," Journal of Neuroinflammation, vol. 15, no. 1 , pp. 39-50, 2018.

[61] H. Hu, Z. Li, X. Zhu, R. Lin, and L. Chen, "Salidroside reduces cell mobility via NF- $\kappa$ B and MAPK signaling in LPS-induced BV2 microglial cells," Evidence-Based Complementary and Alternative Medicine, vol. 2014, Article ID 383821, 8 pages, 2014.

[62] X. Zhang, W. Lai, X. Ying et al., "Salidroside reduces inflammation and brain injury after permanent middle cerebral artery occlusion in rats by regulating $\mathrm{PI} 3 \mathrm{~K} / \mathrm{PKB} / \mathrm{Nrf} 2 / \mathrm{NF} \kappa \mathrm{B}$ signaling rather than complement C3 activity," Inflammation, vol. 42, no. 5, pp. 1830-1842, 2019.

[63] T. Han, "Effects of salidroside pretreatment on expression of tumor necrosis factor-alpha and permeability of blood brain barrier in rat model of focal cerebralischemia-reperfusion injury," Asian Pacific Journal of Tropical Medicine, vol. 6, no. 2, pp. 156-158, 2013.

[64] W. Zuo, F. Yan, B. Zhang, X. Hu, and D. Mei, "Salidroside improves brain ischemic injury by activating PI3K/Akt pathway and reduces complications induced by delayed tPA treatment," European Journal of Pharmacology, vol. 830, pp. 128-138, 2018.

[65] Z. Wei, P. Ming, Y. Yang, X. Zhangwu, S. Bin, and L. Zhaofen, "Protective effects of salidroside on mitochondrial functions against exertional heat stroke-induced organ damage in the rat," Evidence-Based Complementary and Alternative Medicine, vol. 2015, Article ID 504567, 11 pages, 2015.

[66] S. Yu, H. Xu, X. Chi et al., "2-(4-methoxyphenyl) ethyl-2acetamido-2-deoxy- $\beta$-d-pyranoside (a salidroside analog) confers neuroprotection with a wide therapeutic window by regulating local glucose metabolism in a rat model of cerebral ischemic injury," Neuroscience, vol. 391, pp. 60-72, 2018.

[67] S. Yu, Q. Cheng, L. Li, M. Liu, Y. Yang, and F. Ding, "2-(4methoxyphenyl) ethyl-2-acetamido-2-deoxy- $\beta$-d-pyranoside confers neuroprotection in cell and animal models of ischemic stroke through calpain1/PKA/CREB-mediated induction of neuronal glucose transporter 3," Toxicology and Applied Pharmacology, vol. 277, no. 3, pp. 259-269, 2014.

[68] H. Xu, H. Gu, Y. Yang, E. Cai, F. Ding, and S. Yu, "2-(4methoxyphenyl) ethyl-2-acetamido-2-deoxy- $\beta$-d-pyranoside exerts a neuroprotective effect through regulation of energy homeostasis and O-GlcNAcylation," Journal of Molecular Neuroscience, vol. 69, no. 2, pp. 1-11, 2019.

[69] C. Xia, D. Aiqing, Z. Tianqiu, and F. Ding, "Pretreatment with 2-(4-methoxyphenyl) ethyl-2-acetamido-2-deoxy- $\beta$-D-pyranoside attenuates cerebral ischemia/reperfusion-induced injury in vitro and in vivo," PLoS One, vol. 9, no. 7, Article ID e100126, 2014.
[70] D. N. Atochin, G. A. Chernysheva, V. I. Smolyakova et al., "Neuroprotective effects of p-tyrosol after the global cerebral ischemia in rats," Phytomedicine, vol. 23, no. 7, pp. 784-792, 2016.

[71] P. Xu, Y. Wang, W. Sun et al., "Salidroside protects the cardiac function of exhausted rats by inducing Nrf2 expression," Cardiovascular Journal of Africa, vol. 31, no. 1, pp. 25-32, 2020.

[72] Ping, Zheng, Zhang et al., "The protective effects of salidroside from exhaustive exercise-induced heart injury by enhancing the PGC- $1 \alpha-\mathrm{NRF} 1 / \mathrm{NRF} 2$ pathway and mitochondrial respiratory function in rats," Oxidative Medicine \& Cellular Longevity, vol. 2015, Article ID 876825, 9 pages, 2015.

[73] D. Kosanovic, X. Tian, O. Pak et al., "Rhodiola: an ordinary plant or a promising future therapy for pulmonary hypertension? a brief review," Pulmonary Circulation, vol. 3, no. 3, pp. 499-506, 2013.

[74] X. Huang, L. Zou, X. Yu et al., "Salidroside attenuates chronic hypoxia-induced pulmonary hypertension via adenosine A2a receptor related mitochondria-dependent apoptosis pathway," Journal of Molecular and Cellular Cardiology, vol. 82, pp. 153-166, 2015.

[75] C. Chen, Y. Tang, W. Deng, C. Huang, and T. Wu, "Salidroside blocks the proliferation of pulmonary artery smooth muscle cells induced by platelet?derived growth factor-BB," Molecular Medicine Reports, vol. 10, no. 2, pp. 917-922, 2014.

[76] M. Chen, H. Cai, C. Yu et al., "Salidroside exerts protective effects against chronic hypoxia-induced pulmonary arterial hypertension via AMPK $\alpha 1$-dependent pathways," American Journal of Translational Research, vol. 8, no. 1, pp. 12-27, 2016.

[77] Y. G. Ma, J. W. Wang, Y. G. Bai, L. Mei, M.-J. Xie, and Z.-J. Dai, "Salidroside contributes to reducing blood pressure and alleviating cerebrovascular contractile activity in diabetic goto-kakizaki rats by inhibition of L-type calcium channel in smooth muscle cells," BMC Pharmacology and Toxicology, vol. 18, no. 1, pp. 30-45, 2017.

[78] X. L. Wang, X. Wang, L. L. Xiong et al., "Salidroside improves doxorubicin-induced cardiac dysfunction by suppression of excessive oxidative stress and cardiomyocyte apoptosis," Journal of Cardiovascular Pharmacology, vol. 62, no. 6, pp. 512-523, 2013.

[79] H. Zhang, W. S. Shen, C. H. Gao, L.-C. Deng, and D. Shen, "Protective effects of salidroside on epirubicin-induced early left ventricular regional systolic dysfunction in patients with breast cancer," Drugs in ReD, vol. 12, no. 2, pp. 101-106, 2012.

[80] H. Wang, Y. Ding, J. Zhou, X. Sun, and S. Wang, “The in vitro and in vivo antiviral effects of salidroside from Rhodiola rosea L. against coxsackievirus B3," Phytomedicine, vol. 16, no. 2-3, pp. 146-155, 2009.

[81] H. He, X. Chang, J. Gao, L. Zhu, M. Miao, and T. Yan, "Salidroside mitigates sepsis-induced myocarditis in rats by regulating IGF-1/PI3K/Akt/GSK-3 $\beta$ signaling," Inflammation, vol. 38, no. 6, pp. 2178-2184, 2015.

[82] E. P. Daskalopoulos, C. Dufeys, C. Beauloye, L. Bertrand, and S. Horman, "AMPK in cardiovascular diseases," Experientia Supplementum, vol. 107, pp. 179-201, 2016.

[83] J. Wang, F. Lin, L. L. Guo, X.-J. Xiong, and X. Fan, "Cardiovascular disease, mitochondria, and traditional Chinese medicine," Evidence-Based Complementary and Alternative Medicine, vol. 2015, Article ID 143145, p. 7, 2015.

[84] B. M. Hybertson, B. Gao, S. K. Bose, and J. M. McCord, "Oxidative stress in health and disease: the therapeutic potential of Nrf2 activation," Molecular Aspects of Medicine, vol. 32, no. 4-6, pp. 234-246, 2011. 
[85] N. Guo, M. Zhu, X. Han, D. Sui, Y. Wang, and Q. Yang, "The metabolism of salidroside to its aglycone p-tyrosol in rats following the administration of salidroside," PLoS One, vol. 9, no. 8, Article ID e103648, 2014.

[86] O. K. F. Thu, O. Spigset, and B. Hellum, "Noncompetitive inhibition of human CYP2C9 in vitro by a commercial Rhodiola rosea product," Pharmacology Research \& Perspectives, vol. 5, no. 4, Article ID e00324, 2017. 